\title{
Energy Efficient Power Management for 4G Heterogeneous Cellular Networks
}

\author{
Xiang Xu, Gledi Kutrolli and Rudolf Mathar \\ Institute for Theoretical Information Technology, RWTH Aachen University \\ Aachen, Germany 52074 \\ Email: \{xu,kutrolli,mathar\}@ti.rwth-aachen.de
}

\begin{abstract}
Heterogeneous network is a promising solution to improve the energy efficiency of the cellular system. In this work, an energy efficient power management scheme designed for LTE femtocells is presented. Using user and service classification, the proposed algorithm balances the network coverage, average data rate and energy consumption. Simulation results confirms the superiority of proposed algorithm in energy efficiency and coverage.
\end{abstract}

Keywords-Energy efficiency, femtocell, LTE, channel quality indicator

\section{INTRODUCTION}

Towards providing omnipresent connectivity, the mobile cellular networks have experienced a tremendous development and evolved to the 4th generation. However, the fast growing information and communication industry is now a major contributor of $\mathrm{CO}_{2}$ emission [1], which could ultimately cause global warming. Furthermore, along with the depleting fossil fuel resources, the electricity price is escalating. Therefore, to protect the environment and maintain a sustainable development, energy efficiency becomes a critical metric for cellular network design.

One of the key technologies to reduce energy consumption is heterogeneous network, where the cell size is diversified. Small cells (micro/pico/femtocells) can efficiently cover the area with poor signal reception or large data demand. As the latest product of the small cell technology, femtocells are tailored to solve the indoor coverage problem. Modern buildings usually have thick walls and glasses to maintain room temperature and keep away noise. Traditionally, the macro base station, or eNode B (eNB) in LTE terms, needs high transmit (Tx) power in order to penetrate the building walls. Using femtocells, the indoor user equipments (UE) can be served by small indoor base stations. The

This work is partially supported by UMIC, a research project in the frame work of the German excellence initiative. base station in femtocells is often referred as Home eNode B (HeNB). The HeNB typically has a maximum Tx power of $20-30 \mathrm{dBm}$ and covers a range of $10-30$ meters. To exploit the existing infrastructure, the HeNB is generally connected to the backbone network through user's DSL (Digital subscriber line) or cable [2].

Although the heterogeneous network is effective in reducing energy consumption, the total energy used by small cells themselves is increasing. The latest projection shows, that small cells will have more than 70 million shipments by the year of 2017 [3]. Supposing half of them are femtocells and each femtocell requires a power of $12 \mathrm{~W}(105.12 \mathrm{kWh} /$ annum $)$ [4], the total energy consumption of all femtocells will be more than $3.6 \times 10^{9} \mathrm{kWh} /$ annum. With such a large yet growing number, the total energy consumption of femtocells can not be ignored.

Furthermore, the effectiveness of the energy efficient algorithms can not be solely determined by the amount of energy being saved. The purpose of energy efficient algorithms is to save more energy on the premise that the network should maintain its quality of service (QoS) [5]. Since the deployment of femtocells results in new source of interference, the target of the energy efficient strategy is to smartly mitigate the interference and efficiently utilize the spectrum resources.

The majority of the femtocells are meant to be deployed by the users, that means, the femtocells will be in suboptimal locations, with unknown environment and turned on and off randomly. Due to these facts, most of the conventional optimization techniques for macrocells cannot be directly applied to femtocells. An energy efficient spectrum allocation is proposed in [6], to maximize the data rate per unit energy. In [4], according to the user activity detection, the femtocell can be turned into idle mode to save power. An opportunistic power control based on clustering of 


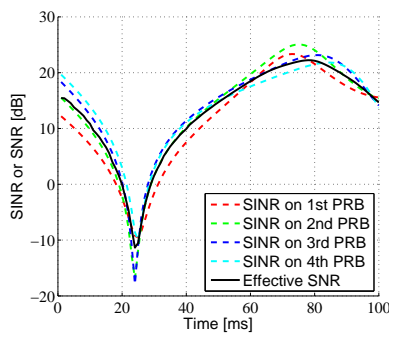

(a)

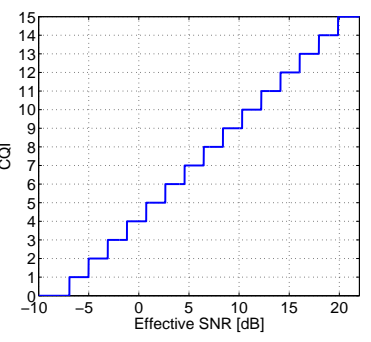

(b)
Fig. 1. (a) SINR to effective SNR mapping. (b) Effective SNR to CQI mapping

the femtocells is proposed in [7]. And in [8], a power control scheme based on measured received (Rx) power of the macro eNB is presented.

In this work, a energy efficient power management scheme for LTE downlink is addressed. The proposed power management scheme takes several realistic constraints into account. Firstly, the communication among eNBs and HeNBs is in coarse time scale, which means, a centralized solution is infeasible. Secondly, the interference mitigation strategies generally require signal to interference plus noise ratio (SINR) as input parameter. However, under current LTE standards, the actual SINR information available at the base station is far from perfect. Therefore, a quantized channel quality indicator (CQI) is adopted as the input of the proposed scheme. Moreover, due to the "user deployed" nature of femtocells, the power control scheme must be autonomous. The proposed power management scheme also considers the QoS for different service types, where the users have different demands.

\section{PRELIMINARIES}

The purpose of this work is to deliver an algorithm which does not depend on strong assumptions and easy to implement in practice. Thus, information about current LTE standards and other related issues is addressed in this section.

\section{A. LTE physical layer}

The LTE downlink is based on orthogonal frequency division multiple access (OFDMA), where information is carried by parallel subcarriers with orthogonal frequencies to overcome frequency selectivity of the wireless channel [9]. In LTE frame structure, a resource element $(\mathrm{RE})$ is defined as 1 subcarrier $\times 1$ OFDMA symbol. A slot, which is usually made up by 7 OFDMA symbols, has a length of $0.5 \mathrm{~ms}$ in time domain. The basic unit for resource allocation is physical resource block (PRB), which consists 12 consecutive subcarriers in 1 slot.

As a feature of OFDMA, different modulation and coding schemes (MCS) can be applied to different PRBs. Moreover, the MCS can be made adaptive to channel conditions, where the base stations can choose either higher data rate or better error protection, according to the channel quality. To facilitate adaptive modulation and coding (AMC), UEs must measure the channel quality and send the information to base stations. To reduce the signaling overhead, the channel quality information is compressed into a 4 bit CQI in LTE standards [10].

The generation of CQI follows two steps. At first, the SINR of several PRBs are compressed into an effective signal to noise ratio (SNR), as depicted in Fig. 1 (a). Transmitting the same amount of information with the effective SNR in additive white Gaussian noise (AWGN) channel will have the same error probability as transmitting with different SINR on different PRBs. The commonly used mapping algorithm is exponential effective SNR mapping (EESM), where the mapping parameters have to be calibrated with extensive simulations [11]. After that, the effective SNR $\gamma$ is mapped into the CQI $Q$ through a step function

$$
Q=f(\gamma),
$$

as shown in Fig. 1. Each CQI value corresponds to a combination of modulation and coding scheme. The MCSs are carefully chosen, such that the block error rate (BLER) does not exceed 10\% in the corresponding channel condition. For the sake of simplicity, the first step of compression is omitted in this work, and the SINR is directly mapped into CQI. It is also assumed that the CQI is available for every PRB.

\section{B. Femtocell deployment}

Due to the orthogonality among subcarriers, there is no inter-channel interference (ICI) in the OFDMAbased LTE networks, assuming perfect frequency synchronization. The only interference left is the cochannel interference (CCI), which exists when signals using the same frequency band overlap.

The multi-tier structure of the heterogeneous network potentially leads to cross-tier interference. If the femtocells use a dedicated frequency band, the crosstier interference can be eliminated [12]. However, the spectrum is an extremely valuable resource, and each operator has its own limited spectrum. Although the cross-tier interference is mitigated, a large amount of macro UE (MUE) must share a reduced spectrum, 
which will eventually cause degradation in macrocell capacity.

In contrast, the more commonly used scheme is partial sharing, where the eNBs can use the whole spectrum and HeNBs can only use a part of the spectrum, since HeNBs typically serve much less UEs. Partial sharing also enables smart spectrum allocation, where the eNBs can reserve the shared part of the spectrum to avoid interference [13].

Regarding the access mode, femtocells can either have open access or closed subscriber group (CSG). In open access mode, all the UEs entering the range of a HeNB will be automatically handed over to the HeNB. Whereas in CSG, only the licensed UE can become home UE (HUE), and the MUE in the range of HeNB are potential victims of CCI. According to the recent market statistics, the absolute majority of the commercially available femtocells are using CSG [14]. Therefore, only CSG is considered in this work.

\section{Channel model}

On top of the conventional co-tier interference among macrocells, several new kinds of interference arises in the considered scenario with partial sharing and CSG, as shown in Tab. I. The HeNB to HUE interference exists, if the femtocells are densely deployed.

Consider a network with $M_{\mathrm{MC}}$ eNBs and $M_{\mathrm{FC}}$ HeNBs, the total number of base station in this network is

$$
M=M_{\mathrm{MC}}+M_{\mathrm{FC}} .
$$

The SINR can be calculated with the Rx power of the signal and interferences. Taking a HUE $n$, which suffers from both co-tier and cross-tier interference, as an example, the SINR can be written as

$$
\gamma_{n}(t)=\frac{P_{m, n}(t)}{\sum_{i=1}^{M_{\mathrm{MC}}} P_{i, n}(t)+\sum_{j=M_{\mathrm{MC}}+1, j \neq m}^{M} P_{j, n}(t)+W_{n}(t)},
$$

where the indices of interfering eNB and HeNB are denoted by $i$ and $j$, respectively. $P_{m, n}$ is the Rx power of the serving HeNB $m . P_{i, n}$ is the Rx power of

\begin{tabular}{c|c|c|c|}
\hline Aggressor & Victim & Type \\
\hline eNB & MUE & Co-tier \\
\hline eNB & HUE & Cross-tier \\
\hline HeNB & MUE & Cross-tier \\
\hline \multirow{4}{*}{ TABLE I. } & HeNB & HUE & Co-tier \\
\cline { 2 - 3 } & Co-TIER AND CROSS-TIER INTERFERENCE TABLE \\
& {$[15]$}
\end{tabular}

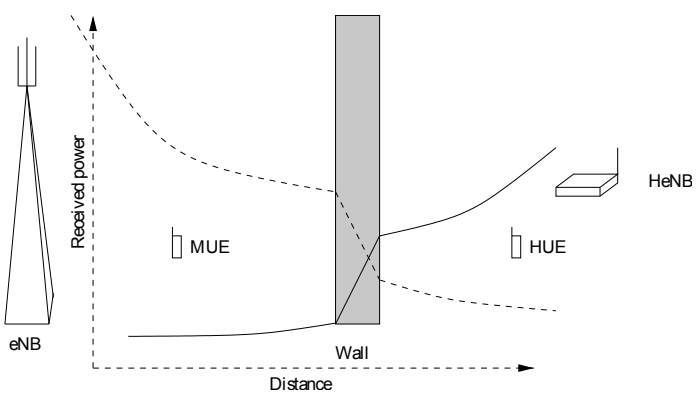

Fig. 2. Rx power in heterogeneous network

the interfering eNB $i$ and $P_{j, n}$ is the Rx power of interfering HeNB $j$, respectively. $W_{n}$ is the thermal noise power and $t$ is the time index. In practice, the femtocells have very small Tx power, thus, many $P_{j, n}$ would be close to zero and neglected. The SINR of other UEs can be calculated similarly.

The Rx power $P_{m, n}$ can be determined by

$$
P_{m, n}=\frac{S_{m}(t) G_{m} H_{m, n}(t)}{L_{m, n}(t)},
$$

where $S_{m}$ is the Tx power, $G_{m}$ is the constant antenna gain, $H_{m, n}$ is the magnitude of the normalized fast fading process and $L_{m, n}$ is the pathloss. The fast fading is usually characterized by the Rayleigh process and can be realized with auto-regressive (AR) filtering [16]. The commonly used model for the pathloss has the general form of

$$
L_{m, n}(t)=A+C \log d_{m, n}(t),
$$

where parameters $A$ and $C$ must be empirically determined according to the radio wave propagation environment and $d_{m, n}$ is the distance between base station $m$ and user $n$. In addition, the wall penetration loss should be applied to the pathloss, as shown in Fig. 2.

\section{Traffic model}

In most of the literature, the system performance is evaluated with channel capacity, which is directly deduced from the SINR using Shannon's formula [12] [17]. However, this traffic model is unrealistic in real systems, especially for users who use voice communications. As long as the QoS requirement is met, the user cannot benefit from extra frequency band or further boost of signal strength.

In this work, the data traffic is modeled in a less abstract level. Three kinds of services are specified, namely, voice over IP (VoIP) service, data service and web service. The VoIP users have the highest priority, which means the frequency resources will be first 
allocated to the VoIP users. The data rate requirement of VoIP users is fixed to $64 \mathrm{kbps}$, in another word, the throughput per unit energy can only be improved by reducing the power margin but not by increasing the data rate. Thus, this kind of user is also called margin adaptive (MA). The data users are also margin adaptive, but their priority is lower than the VoIP users. The data rate requirement for data users is a random number in the range of [512 - 2000] kbps. In contrast, the web users have a minimum data rate requirement, and the data rate should be maximized subject to the power limit. This kind of user is also called rate adaptive (RA) [18]. The assumed distribution of the users with different services is summarized in Tab. II.

\section{POWER MANAGEMENT}

In power management, the HeNBs can adjust their Tx power for higher energy efficiency and avoidance of interference. However, subcarrier level power allocation is still not possible in current LTE standards, only PRB assignment can be performed in the resource allocation procedure.

\section{A. Resource allocation}

The resource allocation scheme should take both efficiency and fairness into account. Due to the frequency selective channels, each UE has different CQIs on different PRBs. The UEs with largest variation in their CQIs are most sensitive in the resource allocation. Therefore, those UEs should choose their PRBs first. The variance is used in this work to measure the variation in CQI.

Suppose there are in total

$$
N_{m}=N_{\mathrm{MA}, m}+N_{\mathrm{RA}, m}
$$

users associated to cell $m$, where $N_{\mathrm{MA}, m}$ is the number of MA users and $N_{\mathrm{RA}, m}$ is the number of RA user, respectively. The users are first sorted according to their service type. For each service type, the users are again sorted according to the variance of their CQIs. The user which has the highest variance pick the PRBs from the available PRB set $\mathcal{P}_{m}$, until its minimum data rate requirement $R_{n}$ is met. And these PRBs are subtracted from set $\mathcal{P}_{m}$. After that, the user with second highest variance will pick. This process goes on iteratively until

\begin{tabular}{|c|c|c|c|}
\hline Service & Priority & Rate requirement & Ratio \\
\hline VoIP & High & $64 \mathrm{kbps}$ & $10 \%$ \\
\hline Data & Mid & {$[512-2000] \mathrm{kbps}$} & $40 \%$ \\
\hline Web & Low & $\geq 64 \mathrm{kbps}$ & $50 \%$ \\
\hline \multicolumn{2}{|c}{ TABLE II. DIFFERENT TYPES OF SERVICE }
\end{tabular}

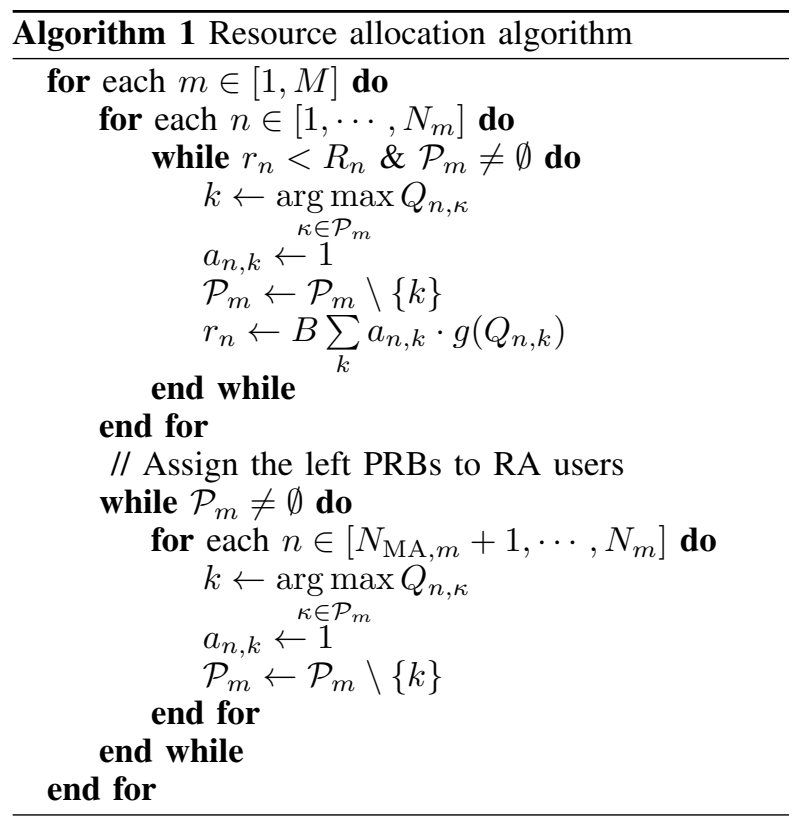

there is no PRB left or all the users have their minimum data rate requirements satisfied. If there are still PRBs left, those PRBs will be assigned to the RA user in the second round. The RA users pick the PRBs one at a time until there is no PRB left. Assuming the users are already sorted, and the user index is in ascending order, the resource allocation algorithm is summarized in Algorithm 1, where $k$ is the index of PRBs, $\emptyset$ is empty set. The assignment parameter $a_{n, k}$ is defined as

$$
a_{n, k}=\left\{\begin{array}{ll}
1 & \text { PRB } k \text { is assigned to user } n \\
0 & \text { otherwise }
\end{array},\right.
$$

which is initially assigned to be 0 . The calculation of $r_{n, k}$ utilizes the CQI to data rate mapping function $g(\cdot)$. And $B$ is the bandwidth of a PRB.

This resource allocation algorithm is used by both eNBs and HeNBs. Since the only information the base stations need to make the resource allocation is the CQIs of their own users, this resource allocation algorithm is completely decentralized.

\section{B. Adaptive power control}

This work focuses on improving energy efficiency of the femtocells, therefore, the macro base stations are assumed to have fixed Tx power. The objective function to achieve energy efficiency is to maximize an utility function associated to the Tx power of HeNBs. Using 


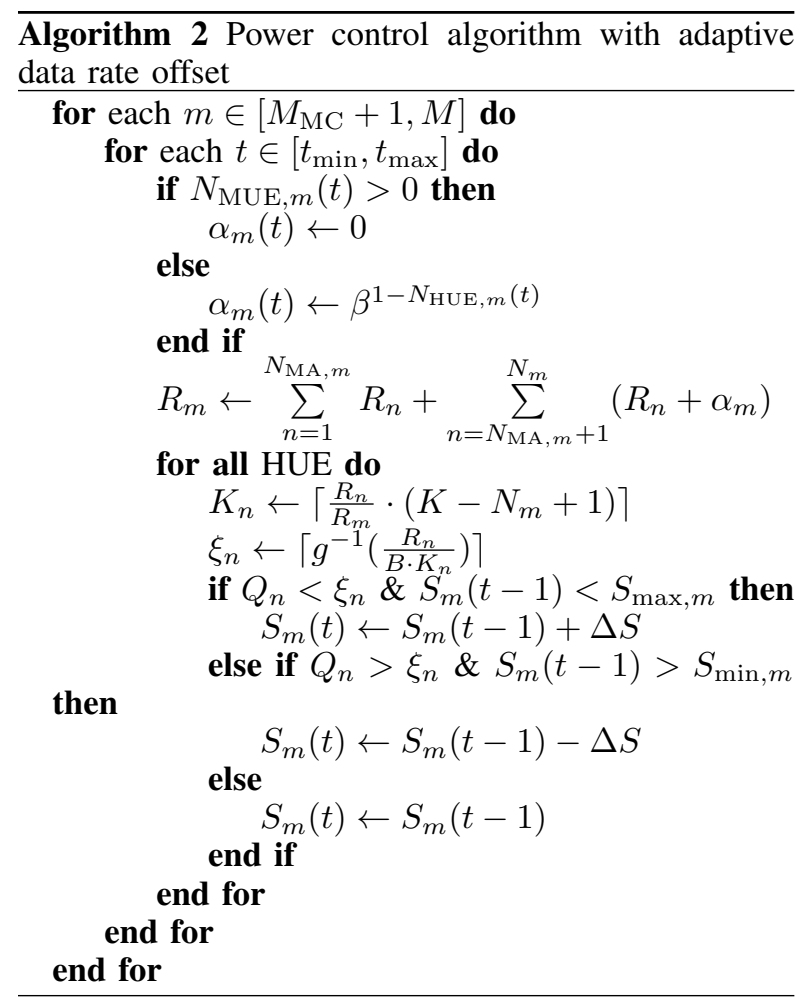

data rate as utility, the utility function can be written as

$$
u(\mathbf{S})=\frac{\sum_{n=1}^{N} r_{n}}{\sum_{m=M_{\mathrm{MC}}+1}^{M} S_{m}},
$$

where $\mathbf{S}=\left[S_{M_{\mathrm{MC}}+1}, S_{M_{\mathrm{MC}}+2}, \cdots, S_{M}\right]$ is the vector of HeNB Tx power. Be aware that adjusting Tx power of HeNBs also affects MUE, because of the interference. The summation of data rate is not only over HUEs, but over all the UEs. The optimal Tx power can be written as

$$
\mathbf{S}_{\mathrm{opt}}=\underset{\mathbf{S} \in \mathcal{S}^{M_{\mathrm{FC}}}}{\arg \max } u(\mathbf{S}),
$$

where $\mathcal{S}$ is the set of feasible Tx power. Clearly, finding optimal solution of this optimization problem requires enormous effort. Moreover, the optimal solution must be centralized. And that implies perfect communications among eNBs and HeNBs, which can not be realized in the current LTE systems. In addition, the data rate based utility function is not the only design criterion. The utility function based on coverage is also very important, and it is also evaluated with simulations in this work.

In this paper, a suboptimal heuristic is adopted. The proposed heuristic works in a totally decentralized fashion. Each HeNB makes its own decision regardless the Tx power of the other HeNBs. Typically, the HeNB needs relatively small Tx power to satisfy the MA users. However, to give RA users the maximum achievable data rate, each HeNB must use much larger Tx power. Therefore, in the proposed algorithm, a data rate offset $\alpha_{m}$ is applied to each RA user upon its minimum data rate demand, after that, the power control algorithm treat the RA users as MA users.

Technically, the RA users are really rate adaptive, only if $\alpha \rightarrow \infty$, whereas the RA users are served with their minimum data rate demand, if $\alpha=0$. However, choosing large $\alpha_{m}$ does not necessarily result in better energy efficiency. The trade-off among capacity, coverage and energy consumption can be observed here [5]. On one hand, lower data rate offset leads to lower capacity in the femtocell. On the other hand, the energy consumption in the femtocell is also lower, thus the energy efficiency is not necessarily worse. Furthermore, lower interference to surrounding MUEs potentially leads to higher macrocell coverage, and HeNBs with overlapped area can also reduce their energy consumption, while maintaining the QoS.

Other than using a fixed value, the data rate offset can be adapted to the number of users in the service range, assuming the HeNB $m$ constantly assesses the number of users in its covered range. Since MUEs, especially indoor MUEs, are most sensitive to interference, the data rate offset is turned to 0 if there is any MUE in bound. Additionally, denote the number of HUEs in the coverage area of HeNB $m$ by $N_{\mathrm{HUE}, m}$. To reduce the interference to HUEs of other HeNBs, $\alpha_{m}$ decreases exponentially as $N_{\mathrm{HUE}, m}$ increases. The base $\beta$ is set to 5 in this work.

As shown in Algorithm 2, the power control algorithm is performed for each HeNB at each time step. After $\alpha_{m}$ is determined, the total data rate demand $R_{m}$ of HeNB $m$ is calculated by summing up the data rate demand of each associated user, while the data rate offset $\alpha_{m}$ is added to each RA user. Then for each associated HUE $n$, its required number of PRB $K_{n}$ is calculated by the proportion of its data rate demand in the total data rate demand. The round up operation guarantees that each user gets at least one PRB and the summation of PRBs over all the HUE does not exceed the total number of available PRBs. After that, a target $\mathrm{CQI} \xi_{n}$ is calculated using the data rate to CQI mapping function $g^{-1}(\cdot)$. If the actual CQI is smaller than the target CQI, the HeNB reduce its Tx power, and vice versa. Notice that the final power control is based on CQI value, even if $\alpha_{m} \rightarrow \infty$, still some power can be 


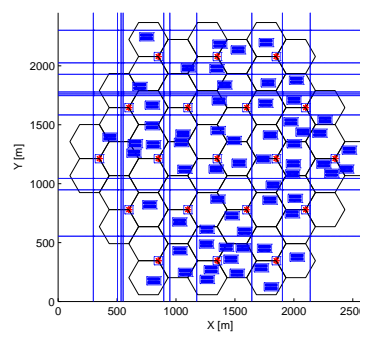

(a)

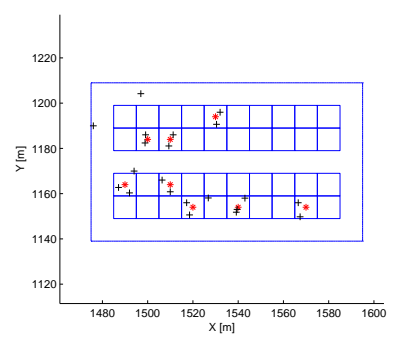

(b)
Fig. 3. (a) Network layout, vertical and horizontal lines indicate streets, boxes indicate buildings, “*”s indicate eNBs. (b) Dual stripe model, "*"s indicate HeNBs, "+" indicate indoor users

saved, because the CQI has a upper limit of 15. And in this case, as soon as the CQI reaches 15 , the power will stop to increase, while the user already has the maximum achievable data rate.

The HUEs are usually indoor and move only with pedestrian speed. Consequently their CQIs vary slowly and can be perfectly predicted [19]. Hence, after the algorithm reaches a steady state, there will seldom be any vibration in the Tx power. The Tx power increment $\Delta S$ determines how fast the algorithm converges. Since CQI is discrete, the variation in SINR $\Delta \gamma$, which changes CQI by 1 , can be calculated using the inverse of (1):

$$
\Delta \gamma=f^{-1}(\Delta Q=1)
$$

Assuming the interference stay unchanged, the CQI can be changed by 1 if the variation in $\mathrm{Rx}$ power $\Delta P$ is the same as $\Delta \gamma$ in $\mathrm{dB}$. Considering there are femtocells with overlapped coverage area, $\Delta S$ is set to $\Delta P / 2$ in this work.

\section{Simulation RESUlts}

\section{A. Simulation environment}

In the evaluation, an urban area with 19 macro sites is simulated. Each macro site serves 3 macro cells, forming a network with 57 macrocells in total. The siteto-site distance is 500 meters. As illustrated in Fig. 3 (a), a number of buildings are randomly located in the simulated area. On average, there is one building per cell. Each building has a structure as shown in Fig. 3 (b), where $10 \times 4$ apartments are within one building [20]. 20\% of the apartments are equipped with HeNBs in the center of the rooms. Half of the HeNBs are turned on at the same time. Some other parameters about the network are summarized in Tab. III.

In total 400 MUEs are located in the simulated area, and $80 \%$ of them are indoor, where each HeNB has 1-5
HUEs placed in the same room. Instead of using static locations, realistic mobility models are implemented for all the UEs. Outdoor users, both pedestrian and vehicular, can only move along certain horizontal and vertical streets, as shown in Fig. 3. Indoor users can only stay indoor and move with relatively low speed. The mobility related parameters are summarized in Tab. IV.

The movements of the users cause time varying Rayleigh fading channel, which is model by AR filtering, whereas the urban environment leads to frequency selectivity, which is modeled by a exponential powerdelay profile [21]. The calculation of pathloss is given in Tab. V, where $\delta$ is the distance between the UE and its projection on the building wall, $q$ is the number of inner walls separating base station and UE, $L_{\mathrm{iw}}=5$ $\mathrm{dB}$ is the inner wall loss and $L_{\mathrm{ow}}=10 \mathrm{~dB}$ is the outer wall loss.

Since the proposed CQI-based power management is aiming at providing low complexity solution which is easy to implement in a real system, a conventional low complexity measurement based power control scheme proposed in [8] is used as reference. The measurement based power control provides the HUE within a radius $d_{\text {max }}$ at least the same amount of Rx power as from the strongest macrocell signal. The Tx power of the measurement based scheme can be written as

$$
S_{m} \triangleq \min \left(P_{m}^{(\mathrm{MC})} L_{m}\left(d_{\max }\right), S_{\max , m}\right),
$$

where $P_{m}^{(\mathrm{MC})}$ is the measured Rx power at the location of HeNB, $L_{m}$ is the femtocell pathloss function. This conventional scheme assumes the HeNB has the ability to measure the Rx power from eNBs. It also heavily relies on the accuracy of the pathloss model.

In the evaluation, the coverage is also compared. The coverage is defined by

$$
\mu=\frac{N_{\text {sat }}}{N_{\text {total }}},
$$

where $N_{\text {sat }}$ is the number of satisfied users, and $N_{\text {total }}$ is the total number of users.

\begin{tabular}{|l|l|l|}
\hline & $\mathrm{HeNB}$ & eNB \\
\hline Carrier frequency & $2 \mathrm{GHz}$ & $2 \mathrm{GHz}$ \\
\hline Spectrum & $2 \mathrm{MHz}$ & $10 \mathrm{MHz}$ \\
\hline Antenna pattern & Omni-directional & 3 -sector \\
\hline Max. Tx power & $20 \mathrm{dBm}$ & $46 \mathrm{dBm}$ \\
\hline Antenna gain & $5 \mathrm{dBi}$ & $14 \mathrm{dBi}$ \\
\hline \multicolumn{2}{|c|}{ TABLE III. } & \multicolumn{2}{|c|}{ SIMULATION PARAMETERS. }
\end{tabular}




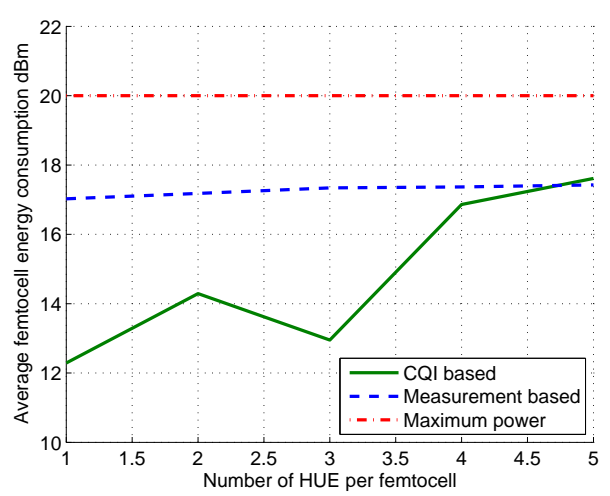

Fig. 4. Average energy consumption of femtocells

\section{B. Numerical results}

Because the trade off of throughput, coverage and energy consumption, the evaluation of energy efficient algorithms is difficult. From Shannon's formula one can conclude that in the ideal case, data rate grows sublinearly to the Tx power, hence, using smaller Tx power gives better throughput the per unit energy [5]. In the real systems, QoS constraints must be taken into consideration. That means, comparing energy efficiency only makes sense, if the power control algorithm can provide enough coverage and throughput. A good power management scheme should be able to find a balance among throughput, coverage and energy efficiency.

The average energy consumption of femtocells is compared for different numbers of HUEs per femtocell in Fig. 4. The measurement based power control gives an average of around $17 \mathrm{dBm}$ Tx power, which is cut the energy consumption by half comparing to no

\begin{tabular}{|c|c|c|}
\hline User & $v_{\text {avg }}$ & Mobility pattern \\
\hline Outdoor pedestrian & $1 \mathrm{~m} / \mathrm{s}$ & Along streets \\
\hline Outdoor vehicular & $10 \mathrm{~m} / \mathrm{s}$ & Along streets \\
\hline Indoor pedestrian & $1 \mathrm{~m} / \mathrm{s}$ & Indoor, random \\
\hline TABLE IV. USER MC & $\begin{array}{l}\text { LITY PAI } \\
\text { LGE SPEE }\end{array}$ & METERS, $v_{\text {avg IS THE }}$ \\
\hline
\end{tabular}

\begin{tabular}{|l|l|}
\hline $\begin{array}{l}\text { eNB - } \\
\text { indoor UE }\end{array}$ & $\begin{array}{l}L_{m, n}=15.3+37.6 \log d_{m, n} \\
+q \cdot L_{\mathrm{iw}}+L_{\mathrm{ow}}\end{array}$ \\
\hline $\begin{array}{l}\text { eNB - } \\
\text { outdoor UE }\end{array}$ & $L_{m, n}=15.3+37.6 \log d_{m, n}$ \\
\hline $\begin{array}{l}\text { HeNB - } \\
\text { indoor UE }\end{array}$ & $\begin{array}{l}L_{m, n}=\max \left(38.46+20 \log d_{m, n},\right. \\
\left.15.3+37.6 \log d_{m, n}\right)+0.7 \delta_{m, n} \\
+q \cdot L_{\mathrm{iw}}\end{array}$ \\
\hline $\begin{array}{l}\text { HeNB - } \\
\text { outdoor UE }\end{array}$ & $\begin{array}{l}L_{m, n}=\max \left(38.46+20 \log d_{m, n},\right. \\
\left.15.3+37.6 \log d_{m, n}\right)+0.7 \delta_{m, n} \\
+q \cdot L_{\mathrm{iw}}+L_{\mathrm{ow}}\end{array}$ \\
\hline
\end{tabular}

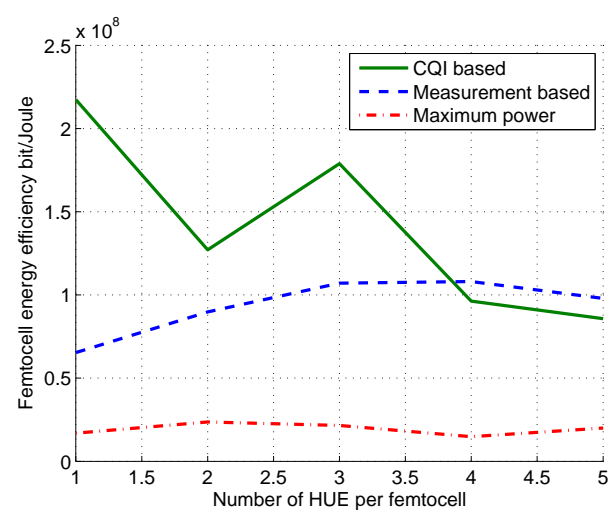

Fig. 5. Average femtocell energy efficiency

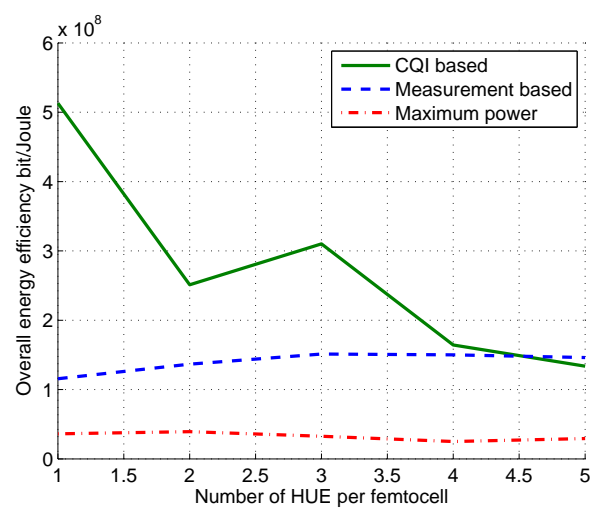

Fig. 6. Average overall energy efficiency

power control. Using the proposed CQI based power control, the energy consumption is even lower for smaller number of users. With 5 HUE per femtocell, the CQI based algorithm offers nearly the same result as the measurement based algorithm.

In Fig. 5, the improvement of bit/Joule energy efficiency of the femtocell using smart power management can be observed. The measurement based algorithm improves the energy efficiency by a large factor comparing to without power control. For smaller number of HUE per femtocell, the advantage of the proposed method is evident. With 1 HUE per femtocell, the energy efficiency is almost three times higher than the measurement based algorithm. The gap closes when there are more HUE per femtocell, no improvement can be seen for 4-5 HUE per femtocell.

Although the throughput of macrocells is not directly generated by femtocells, it heavily depends on the Tx power of femtocells, due to interference. In Fig. 6, the overall throughput per femtocell Tx power is compared. In this figure, the superiority of the proposed algorithm is more clear. By properly managing interference, a fac- 


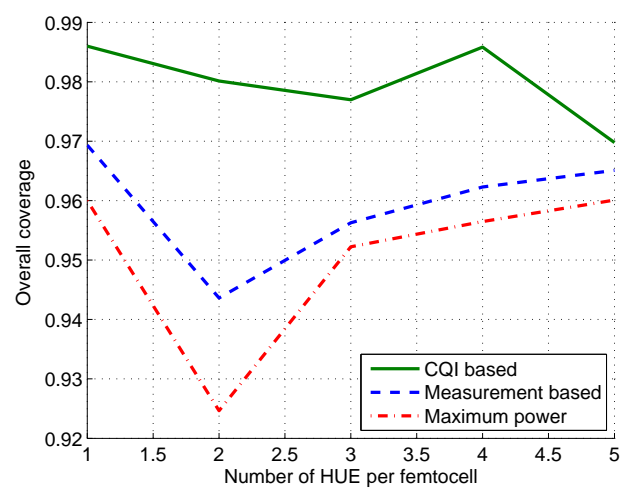

Fig. 7. Average overall coverage

tor of 5 in energy efficiency can be achieved comparing to the measurement based algorithm. And even with larger number of users, there is only a small loss.

Furthermore, the overall coverage of the network is evaluated in Fig. 7, where the proposed CQI based algorithm shows the best performance. As a conclusion, even though the proposed algorithm has slightly worse energy efficiency than the conventional measurement based algorithm for 5 HUEs per femtocell, it offers better coverage for the whole network. In this sense, the proposed power management scheme has the better overall performance than the conventional scheme.

\section{CONCLUSION}

In this work, an energy efficient power management scheme is proposed for LTE femtocells. The proposed scheme takes the QoS constraints as design criteria. The energy efficiency of the proposed scheme is much higher than the conventional scheme with less HUEs per femtocell and comparable to the conventional scheme when there are more HUEs per femtocell. In addition, the proposed scheme offers better coverage. And it is easy to implement in practice, due to the lack of strong assumptions.

\section{REFERENCES}

[1] The Climate Group, Glebe e-Sustainability Initiative (GeSI), "Smart 2020: Enabling the low carbon economy in the information age." 2008. [Online]. Available: http://www.smart2020.org/publications/

[2] Nomor Research, "LTE Home Node Bs and its enhancements in release 9," White paper, May 2010.

[3] Small Cell Forum, "Small cell market status," Feb 2013. [Online]. Available: http://www.smallcellforum.org/

[4] I. Ashraf, L. Ho, and H. Claussen, "Improving energy efficiency of femtocell base stations via user activity detection," in Wireless Communications and Networking Conference (WCNC), IEEE, Sydney, Australia, Apr. 2010.
[5] Y. Chen, S. Zhang, S. Xu, and G. Li, "Fundamental tradeoffs on green wireless networks," Communications Magazine, IEEE, vol. 49, no. 6, pp. 30-37, 2011.

[6] W. Cheng, H. Zhang, L. Zhao, and Y. Li, "Energy efficient spectrum allocation for green radio in two-tier cellular networks," in Global Telecommunications Conference (GLOBECOM), IEEE, Miami, USA, Dec 2010.

[7] M. S. Jin, S. A. Chae, and D. I. Kim, "Per cluster based opportunistic power control for heterogeneous networks," in Vehicular Technology Conference (VTC Spring), IEEE 73rd, Budapest, Hungary, May 2011.

[8] H. Claussen, L. T. W. Ho, and L. G. Samuel, "Self-optimization of coverage for femtocell deployments," in Wireless Telecommunications Symposium, (WTS), Pomona, USA, Apr. 2008.

[9] H. Holma and A. Toskala, LTE for UMTS - OFDMA and SCFDMA Based Radio Access. Wiley, 2009.

[10] 3GPP TS 36.213 V8.8.0, "Evolved universal terrestrial radio access (E-UTRA): Physical layer procedures," Sep. 2009.

[11] S. N. Donthi and N. B. Mehta, "An accurate model for EESM and its application to analysis of CQI feedback schemes and scheduling in LTE," Wireless Communications, IEEE Transactions on, vol. 10, no. 10, pp. 3436-3448, Oct. 2011.

[12] H. Mahmoud and I. Guvenc, "A comparative study of different deployment modes for femtocell networks," in Personal, Indoor and Mobile Radio Communications, IEEE 20th International Symposium on (PIMRC), Tokyo, Japan, Sep. 2009.

[13] A. Engels, M. Reyer, X. Xu, R. Mathar, J. Zhang, and H. Zhuang, "Autonomous self-optimization of coverage and capacity in LTE cellular networks," IEEE Transactions on Vehicular Technology, vol. 62, no. 5, pp. 1989-2004, 2013.

[14] Infonetics Research, "Femtocells up 66\% in 4Q12: big 2013 expected as 4G femtos ramp," Mar 2013. [Online]. Available: http://www.infonetics.com/

[15] N. Saquib, E. Hossain, L. B. Le, and D. I. Kim, "Interference management in OFDMA femtocell networks: issues and approaches," Wireless Communications, IEEE, vol. 19, no. 3, pp. 86-95, 2012.

[16] K. E. Baddour and N. C. Beaulieu, "Autoregressive modeling for fading channel simulation," Wireless Communications, IEEE Transactions on, vol. 4, no. 4, pp. 1650-1662, Jul. 2005.

[17] Z. Lu, Y. Sun, X. Wen, T. Su, and D. Ling, "An energy-efficient power control algorithm in femtocell networks," in Computer Science Education (ICCSE), 7th International Conference on, Melbourne, Australia, Jul 2012.

[18] C. Liu, A. Schmeink, and R. Mathar, "Dual optimal resource allocation for heterogeneous transmission in OFDMA systems," in Globecom, IEEE, Honolulu, Hawaii, USA, Dec. 2009.

[19] M. Ni, X. Xu, and R. Mathar, "A channel feedback model with robust SINR prediction for LTE systems," in The 7th European conference on antennas and propatation (EuCAP), Gothenburg, Sweden, Apr 2013.

[20] 3GPP R4-092042, "Simulation assumptions and parameters for FDD HeNB RF requirements," Alcatel-Lucent, picoChip Designs, Vodafone.

[21] X. Xu and R. Mathar, "Low complexity joint channel estimation and decoding for LDPC coded MIMO-OFDM systems," in Vehicular Technology Conference (VTC Spring), IEEE 73rd, Budapest, Hungary, May 2011. 